\title{
PERFIL DAS AFECÇÕES DEMANDADAS PARA REABILITAÇÃO PELO SISTEMA ÚNICO DE SAÚDE NOS SERVIÇOS CREDENCIADOS DE UM MUNICÍPIO DO EXTREMO SUL CATARINENSE
}

\author{
Profile of the defendants disorders for rehabilitation by the Unified Health \\ System in accredited services of a municipality's in the Extreme South of Santa \\ Catarina
}

Nathiele Placido Belettini ${ }^{1}$, Franciani Rodrigues da Rocha ${ }^{2}$, Lisiane Tuon ${ }^{3}$, Willians Cassiano Longen ${ }^{4}$

${ }^{1}$ Fisioterapeuta. Pós-Graduada em Fisioterapia Traumato Ortopédica e Esportiva UNESC.

2 Fisioterapeuta. Pós-Graduada em Residência Multiprofissional em Saúde. Mestranda do Programa de Pós-Graduação em Ciências da Saúde PPGCS/UNESC.

3 Fisioterapeuta. Doutora em Ciências da Saúde - PUC/RS. Professora e Pesquisadora do Programa de Pós-Graduação em Saúde Coletiva PPGSCoI/UNESC.

4 Fisioterapeuta. Doutor em Ciências da Saúde - PPGCS/UNESC. Professor e Pesquisador do Programa de Pós-Graduação em Saúde Coletiva IPPGSCoI/UNESC.

\section{Endereço para correspondência:}

Willians Cassiano Longen

Programa de Pós-Graduação em Saúde Coletiva - PPGSCol

Universidade do Extremo Sul Catarinense - UNESC

Av. Universitária, 1105 - Universitário - Criciúma/SC

CEP: 88806-000 C.P: 3167

Email:wcl@unesc.net 


\title{
Resumo
}

Com a criação do SUS a Fisioterapia passa a integrar-se ao sistema, sendo um serviço de especialidade de direito do cidadão, considerada como um procedimento de média complexidade dentro do SUS e responsável por uma parte dos recursos públicos destinados ao financiamento da saúde. De modo geral no país, a atuação ambulatorial de Fisioterapia no SUS vem aumentando consideravelmente. O objetivo do estudo é identificar a demanda da Fisioterapia regulada para atendimento no SUS no Município de Criciúma-SC. Caracteriza-se como transversal, qualiquantitativo, levantamento de dados, exploratório e descritivo. Em Criciúma existe a atenção especializada, dentre estas está a Fisioterapia, a qual possui um setor próprio de cadastro do paciente, verificação da requisição e autorização da mesma. Os dados foram coletados no setor de autorização de Fisioterapia, no período de janeiro a dezembro de 2012. Para estatística utilizou-se o software de estatística SPSS versão 18.0. Houve prevalência dos acometimentos ortopédicos, neurológicos e traumatológicos. O sexo feminino se destacou na área de ortopedia, reumatologia e uroginecologia, já o masculino foi na traumatologia, cardiorrespiratória e neurológica. Mostra-se a necessidade de articulação em rede com as políticas públicas de prevenção e promoção da saúde do usuário.

Palavras-chave: Fisioterapia; SUS; Média complexidade; Reabilitação.

\begin{abstract}
With the creation of SUS physical therapy becomes part of the system being a specialty service of a citizen's right, considered as a procedure of medium complexity within the SUS and is responsible for a portion of public funds intended for the financing of health. Generally in the country the performance physiotherapy ambulatory in the SUS is increasing considerably. The objective of this study is to identify the regulated physiotherapy to meet demand in the SUS in the municipality of Orestiada. is characterised as transversal, quali-quantitative survey data, exploratory and descriptive. There is specialized attention in Criciúma, tooth these is physical therapy, which has an own sector patient registration, verification of the request and authorization of the same, the data were collected in the sector of physiotherapy and authorization in the period January to December 2012. For statistics using the
\end{abstract}




\section{Artigo Original}

\section{Saúde Funcional}

statistical software SPSS version 18.0. There was the prevalence Orthopedic, neurological and performing different traumatológicos. The female gender excelled in orthopedics, rheumatology and urogynecology, the male was in trauma, neurological and cardiorespiratory. Shows the need for networking with the public policies of prevention and promotion of health of the user.

Keywords: Physiotherapy; SUS; Medium Complexity.

\section{INTRODUÇÃO}

Até a metade do século XX no Brasil era priorizada a assistência curativa no atendimento médico individualizado. Porém, para melhorar e expandir os atendimentos oferecidos à população carente, fez-se com que fossem revistos os modelos adotados até então na área da saúde ${ }^{1-5}$.

Em 1990 criou-se o Sistema Único de Saúde (SUS), com um conceito novo do método saúde-doença, visando um conjunto de ações em saúde que abrangem um ciclo completo e integrado entre a promoção, prevenção e recuperação da saúde. A Lei 8.080/1990 que regulamenta o SUS traz no Artigo 2o: "a saúde é um direito fundamental do ser humano, devendo o Estado prover as condições indispensáveis ao seu pleno exercício"1.

O SUS tem como princípios: Universalização, Integralidade, Igualdade, Descentralização, Hierarquização e Participação Popular ${ }^{1,5}$. A criação e implantação gradativa do SUS podem ser consideradas como uma das reformas sociais mais importantes ocorridas no Brasil e no mundo ${ }^{6}$.

Diante da criação do SUS, a Fisioterapia passa a integrar-se ao sistema sendo um serviço de especialidade de direito do cidadão. A Fisioterapia é uma ciência da Saúde que estuda, previne e trata os distúrbios cinéticos funcionais, intercorrentes em órgãos e sistemas do corpo humano, gerados por alterações genéticas, por traumas e por doenças adquiridas. Diante disto o profissional fisioterapeuta tem total capacidade para atuar em todos os âmbitos de Atenção a Saúde, contribuindo para a eficiência da mesma. Com o passar do tempo a Fisioterapia adquiriu crescente importância nos serviços de Atenção a Saúde, pois 


\section{Artigo Original}

\section{Saúde Funcional}

vem desenvolvendo suas habilidades na prevenção, promoção e reabilitação nos âmbitos individuais e coletivos ${ }^{5,7-9}$.

A Fisioterapia como ciência é transferida socialmente como procedimento de média complexidade dentro do sistema e é responsável por parte dos recursos públicos destinados ao financiamento da saúde. De modo geral no país, a produção ambulatorial de Fisioterapia no SUS vem aumentando consideravelmente, consequentemente os custos também aumentaram. Torna-se assim de fundamental importância reflexões acerca não apenas dos procedimentos e técnicas que os envolvem, mas em paralelo sobre o contexto amplo de saúde que discorrem à necessidade de recuperação com a Fisioterapia, para que se tenha uma ação estruturada levando a um resultado mais fidedigno da atividade ${ }^{10}$.

O município de Criciúma é o maior do Sul Catarinense e um dos cinco maiores de Santa Catarina, possui 235.709 km² e a população estimada em 2013 é de 202.39511. Na saúde pública a cidade de Criciúma possui a atenção especializada, a qual funciona através de encaminhamentos, estes na sua grande maioria originada da atenção básica. Dentre as especialidades encontradas no município está a Fisioterapia, a qual possui um setor próprio de autorização desde 2012, o qual realiza o cadastro do paciente, verifica autenticidade da requisição e autoriza a mesma e encaminha o usuário a uma clínica municipal ou conveniada. Para isso o usuário deve ir a este setor munido de prescrição médica, conforme normativa interna da Secretaria Municipal do Sistema de Saúde. O setor possui controles próprios, entre eles o controle mensal, o qual possui, dentre os dados, gênero, diagnóstico clínico e idade ${ }^{12}$.

Evidenciou-se ser necessária a realização desta pesquisa para que através dela possa se identificar a real demanda da Fisioterapia no SUS no Município de Criciúma-SC, delimitando os diagnósticos clínicos mais prevalentes e o gênero mais afetado, pois assim torna-se possível ao município o desenvolvimento de ações preventivas na Atenção à Saúde.

\section{METODOLOGIA}

Esta pesquisa foi um estudo quali-quantitativo, de levantamento de dados, exploratório e descritivo. 
Foi solicitada autorização para realização da presente pesquisa à Secretaria Municipal do Sistema de Saúde de Criciúma através do protocolo no 391986, a qual foi deferida.

Os dados foram coletados no Setor de Autorização de Fisioterapia do Município de Criciúma, que possui o controle mensal de autorizações. Nestes foram coletados os dados de idade, gênero e diagnóstico clínico, no período entre janeiro e dezembro de 2012.

Os dados coletados foram devidamente tabulados, analisados e avaliados pelo programa de estatística SPSS 18.0 para Windows, utilizando a Análise de Frequências para análise das porcentagens, média, mínimo, máximo e desvio padrão (DP). Após os resultados foram confirmados através do Teste Qui-Quadrado de Pearson para frequência das variáveis categóricas binomiais. Foram considerados como estatisticamente significativos os resultados que obtiveram $p<0,05$. Após realizados os testes estatísticos, os dados foram transferidos ao software Microsoft Word para a organização das tabelas e, assim, serem confrontados com a literatura científica.

\section{RESULTADOS}

A média de requisições para a realização de Fisioterapia entre os meses de janeiro a dezembro de 2012 foi de $418,33 \pm 79,28$. O maior número de requisições foi no mês de agosto, com 555 requisições, e o menor em dezembro de 2012, com 265 requisições. Ao total, no ano de 2012 foram 5020 requisições autorizadas (Figura 1). 


\section{Artigo Original}

\section{Saúde Funcional}

Figura 1. Requisições entre os meses de Janeiro e Dezembro de 2012. Distribuição mês a mês do quantitativo de requisições recebidas pelo Setor de Regulação da Secretaria Municipal de Saúde.

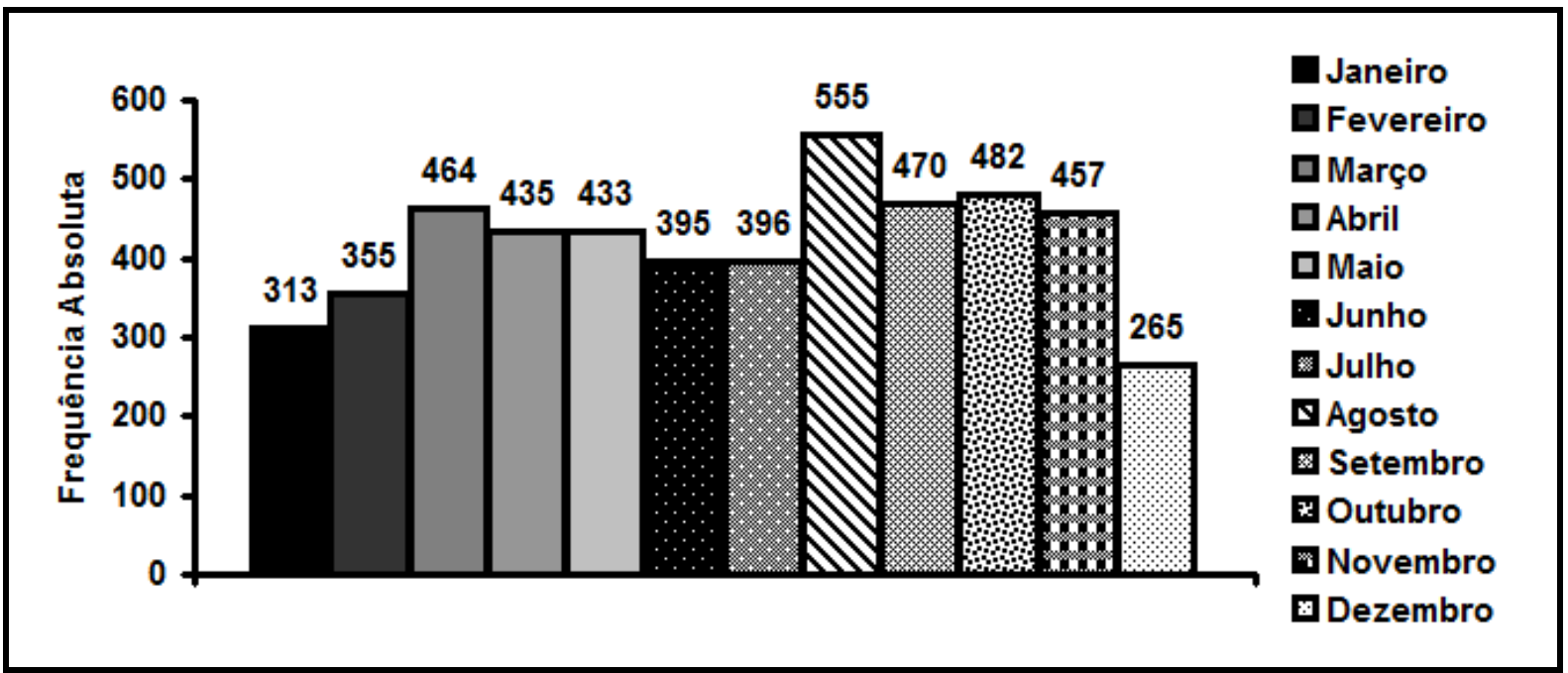

Fonte: Próprio Autor

Dentre os diagnósticos que predominaram encontrou-se a Ortopedia, com $41,7 \%$, em segundo a Neurologia, com $20,3 \%$, que foi seguida pela Traumatologia, com 16,2\% (Tabela 1).

Tabela 1. Distribuição Quanto à Especialidade Envolvida no Diagnóstico. Especialidades relacionadas aos diagnósticos estabelecidos nas requisições médicas recebidas pelo Setor de Regulação da SMS.

\begin{tabular}{ccc}
\hline Diagnóstico & $\mathbf{N}$ & $\%$ \\
\hline Ortopedia & 2091 & 41,7 \\
Neurologia & 1018 & 20,3 \\
Traumatologia & 811 & 16,2 \\
Sem Diagnóstico & 604 & 12,0 \\
Reumatologia & 235 & 4,7 \\
Outros & 117 & 2,3 \\
Cardiorespiratória & 78 & 1,6 \\
Uroginecologia & 66 & 1,3 \\
TOTAL & $\mathbf{5 0 2 0}$ & $\mathbf{1 0 0 , 0}$
\end{tabular}

Fonte: Próprio Autor 
Artigo Original

Saúde Funcional

Dentre o gênero, o feminino se destacou com $57,4 \%$ do total e o masculino ficou em 42,6\% (Tabela 2).

Tabela 2. Distribuição em Relação ao Sexo dos Usuários. Separação da totalidade dos casos regulados pelo sexo.

\begin{tabular}{ccc}
\hline SEXO & $\mathbf{n}$ & $\%$ \\
\hline Feminino & 2879 & 57,4 \\
Masculino & 2141 & 42,6 \\
TOTAL & 5020 & $\mathbf{1 0 0 , 0}$ \\
\hline
\end{tabular}

Em relação à Associação entre o Gênero e o Diagnóstico apresentado, observa-se nesta pesquisa que o gênero feminino buscou mais atendimento nas áreas de Ortopedia, com 1384 de 2091 requisições, 74 de outras patologias de 117 desta classificação, 166 na reumatologia das 235 requisições, 327 mulheres buscaram atendimento sem diagnóstico descrito entre 604 requisições com a mesma classificação, 62 apresentavam como diagnóstico na área de uroginecologia dentre 66 requisições. O Gênero masculino teve maior busca nas requisições de neurologia, com 568 entre 1018 requisições, 431 em traumatologia, no total de 811 desta área, e cardiorrespiratória com 42 requisições de 78 desta área (Tabela 3).

Tabela 3. Associação entre o Gênero e o Diagnóstico Apresentado. Método Estatístico Empregado: Teste Qui-Quadrado de Pearson $(p<0,05)$.

\begin{tabular}{cccc}
\hline DIAGNÓSTICO & \multicolumn{2}{c}{ SEXO } & TOTAL \\
\cline { 2 - 4 } Cardiorespiratória & Feminino & Masculino & \\
Neurologia & 36 & 42 & 78 \\
Ortopedia & 450 & 568 & 1018 \\
Outros & 1384 & 707 & 2091 \\
Reumatologia & 74 & 43 & 117 \\
Sem Diagnóstico & 166 & 69 & 235 \\
Traumatologia & 327 & 277 & 604 \\
Uroginecologia & 380 & 431 & 811 \\
TOTAL & 62 & 4 & 66 \\
& $\mathbf{2 8 7 9}$ & $\mathbf{2 1 4 1}$ & $\mathbf{5 0 2 0}$
\end{tabular}

Fonte: Próprio Autor 


\section{DISCUSSÃO}

Segundo Aleluia e Santos ${ }^{10}$, houve um aumento expressivo na produção ambulatorial de Fisioterapia no SUS no Município de Salvador, Bahia, de 1995-2007, o que está diretamente relacionado com o aumento do custo também. Destacaram ainda que em 2002, no Brasil, quando se comparou o custo com Fisioterapia, foi observado um gasto significativo em diversos estados do país.

No presente estudo foi possível observar que a demanda de Fisioterapia no Município de Criciúma foi de 5020 requisições autorizadas em 2012. Em relação à prevalência do diagnóstico foi observado o destaque com $41,7 \%$ ortopedia, $20,3 \%$ neurologia e 16,3\% traumatologia, o que confere com os achados da literatura.

Felix et al. ${ }^{13}$, em sua pesquisa com 0 objetivo de descrever 0 perfil epidemiológico dos pacientes atendidos na Clínica Escola de Fisioterapia da Universidade Estadual de Goiás, teve como resultados $72,8 \%$ dos pacientes da área musculoesquelética e $34,8 \%$ da área neurofuncional.

A procura da população por atendimento médico e de Fisioterapia devido aos distúrbios do sistema musculoesquelético estão entre os maiores motivos de procura. Relatam que o aumento da prática esportiva colabora para a incidência de lesões e que os trabalhadores passam a apresentar diversas lesões musculoesqueléticas decorrentes da sua prática laboral ${ }^{14}$.

Em relação ao predomínio do gênero na população brasileira, as mulheres formam um conjunto maior do que os homens, isso ocorre devido a maior expectativa de vida das mesmas e também a busca pelos serviços de saúde ${ }^{15}$. Da população total brasileira, $50,1 \%$ são mulheres, e o restante, $49,9 \%$, homens ${ }^{16}$, sendo que na amostra houve predomínio do gênero feminino com $57,4 \%$, enquanto o masculino $42,6 \%$. Em um estudo visando o perfil epidemiológico dos pacientes atendidos em uma clínica escola de Fisioterapia da Universidade Estadual de Góias foi observado que, dos 129 pacientes, 45 eram do sexo masculino enquanto 83 eram do sexo feminino ${ }^{13}$.

Em relação ao gênero e ao diagnóstico, o feminino teve uma prevalência nas áreas de ortopedia, reumatologia e uroginecologia. Oliveira e Braga ${ }^{14}$, em seu estudo, com o objetivo de descrever o perfil dos pacientes atendidos na Clínica de Fisioterapia aplicada à ortopedia e traumatologia da Universidade Paulista, relatam 


\section{Artigo Original}

\section{Saúde Funcional}

que o sexo feminino está vinculado a um risco maior de doenças, porém os motivos ainda são controversos, mas o mais estudado atualmente é a carga hormonal. Eles tiveram como resultados de seu estudo a incidência de $62,37 \%$ nas mulheres.

Em outra pesquisa que avaliou o perfil epidemiológico dos pacientes atendidos no Serviço de Fisioterapia Reumatológica da Faculdade de Educação Física e Fisioterapia da Universidade de Passo Fundo, o sexo feminino prevaleceu com 210 pacientes $(85,4 \%)$ contra $14,6 \%$ do sexo masculino ${ }^{17}$.

O estudo que objetivava a análise do perfil do serviço de Fisioterapia do setor secundário do SUS de Juiz de Fora/MG teve como resultado a prevalência das mulheres na área de reumatologia e ortopedia ${ }^{18}$.

As mulheres, devido suas diferenças anatômicas, alterações hormonais, gestação e partos, são mais favorecidas a terem mais problemas relacionados ao assoalho pélvico do que os homens ${ }^{19}$. Esta pesquisa vem confirmar os achados, pois foram as mulheres que mais buscaram os atendimentos em uroginecologia.

Cardiorrespiratória, neurologia e traumatologia foram as especialidades que predominaram no gênero masculino. Barbosa et al..$^{8}$ destacam em seus resultados a prevalência do sexo masculino nas lesões traumatológicas de membros superiores, onde, dos 223 avaliados, 116 eram homens, $62,50 \%$.

Em uma pesquisa que analisou o perfil funcional de pacientes com doenças neurológicas que se encontravam em tratamento fisioterapêutico no Centro de Promoção e Reabilitação em Saúde e Integração Social (PROMOVE São Camilo), $61,29 \%$ eram do sexo masculino ${ }^{20}$.

Outra pesquisa aponta que $72,09 \%$ da amostra de portadores de acidente vascular encefálico era composta por homens ${ }^{21}$. No estudo para avaliar o perfil dos pacientes frequentadores da Clínica de Fisioterapia Pneumofuncional da Universidade do Vale do Paraíba, $56 \%$ dos pacientes eram do sexo masculino ${ }^{22}$.

Santana et al. ${ }^{23}$, traz em seu estudo que de 75 pacientes, 40 eram do gênero masculino. $O$ objetivo do estudo era analisar o perfil dos pacientes atendidos no setor de Fisioterapia Respiratória do Centro de Saúde da UNIT, no período de 2007 a 2008. 


\section{CONCLUSÃO}

$\mathrm{Na}$ amostra foi possível identificar a demanda de Fisioterapia no SUS, no qual prevaleceram os acometimentos ortopédicos, neurológicos e traumatológicos. O gênero feminino se destacou na área de ortopedia, reumatologia e uroginecologia, já o masculino foi na traumatologia, cardiorrespiratória e neurológica.

Diante da demanda de Fisioterapia, torna-se importante o olhar voltado à atenção básica, sendo possível ter como foco a promoção e prevenção, visando amenizar a demanda acentuada para a Fisioterapia para a reabilitação e assim tentar melhorar a qualidade de vida dos usuários do SUS. Mostra-se a necessidade de articulação em rede com as políticas públicas de prevenção e promoção da saúde do usuário.

\section{REFERÊNCIAS}

1. Berti PM. O Sistema Único de Saúde-SUS-sua criação, seus princípios e a efetivação do direito à saúde [TCC]. Santa Rosa: Universidade Regional do Noroeste do Estado do Rio Grande do Sul; 2012.

2. Buss PM, Carvalho Al. Desenvolvimento da promoção da saúde no Brasil nos últimos vinte anos (1988-2008). Ciênc saúde coletiva. 2009;14(6):2305-16.

3. Ragasson CAP, Almeida DCS, Comparin K, Mischiati MF, Gomes JT. Atribuições do fisioterapeuta no programa de saúde da família: reflexões a partir da prática profissional. Olho mágico. 2005;13(2):1-8.

4. Trelha CS, Silva DW, Lida LM, Fortes MH, Mendes TS. O fisioterapeuta no programa de saúde da família em Londrina (PR). Espaç Saude. 2007;8(2):20-5.

5. Veiga AC, Neves CAS, Montagna P, Kanda SS, Valença SS. A atuação do fisioterapeuta na unidade básica de saúde. Fisioter Bras. 2004;5(3):246-49.

6. Brasil. Ministério da Saúde. A construção do SUS; 2006.

7. Baraúna MA, Testa CEA, Guimarães ÉA, Boaventura CM, Dias AL, Strini PJSA, et al. A importância da inclusão do fisioterapeuta no Programa de Saúde da Família. Fisioter Bras. 2008;9(1):64-9.

8. Barbosa RI, Raimundo KC, Fonseca MCR, Coelho DM, Ferreira AM, Hussein AM, et al. Perfil dos pacientes com lesões traumáticas do membro superior atendidos pela fisioterapia em hospital do nível terciário. Acta fisiátrica. 2013;20(1):14-9. 


\section{Artigo Original}

\section{Saúde Funcional}

9. Castro SS, Cipriano GJ, Martinho A. Fisioterapia no programa de saúde da família: uma revisão e discussões sobre a inclusão. Fisioter mov. 2006;19(4):55-62.

10. Aleluia ÍRS, Santos FC. Analise dos auditores em saúde quanto aos serviços públicos de Fisioterapia no Estado da Bahia. Revista Eletrônica Gestão \& Saúde. 2013;4(1):1499-515.

11. IBGE. Contagem final da População 2013; 2013.

12. Secretaria Municipal da Saúde de Santa Catarina. Implantação de políticas para controle, avaliação e auditoria dos serviços de Fisioterapia do Município de Criciúma/SC, 2011.

13. Felix JF, Rezende LA, Ferreira VC, Formiga CKMR. Perfil epidemiológico dos pacientes tratados na clínica escola de fisioterapia da UEG. Anais do IX Seminário de Iniciação Científica VI Jornada de Pesquisa e Pós-Graduação e Semana Nacional de Ciência e Tecnologia, Universidade Estadual de Goiás. 2011;19.

14. Oliveira AC, Braga DLC. Perfil epidemiológico dos pacientes atendidos na clínica de ortopedia da Universidade Paulista. J Health Sci Inst. 2010;28(4):356-8.

15. Fabrício SCC, Wehbe G, Nassur FB, Andrade Jl. Assistência domiciliar: a experiência de um hospital privado do interior paulista. Rev Latino-Am Enfermagem. 2004;12(5):721-6.

16. IBGE. Contagem final da População 2007; 2007.

17. Wibelinger LM, Tombini DK. Perfil epidemiológico dos pacientes atendidos no serviço de fisioterapia reumatológica da Universidade de Passo Fundo. Rev Bras de Ciên do Envelh Hum. 2012;7(2).

18. Machado GLR, Fayer VA, Caldas MAJ, Cruz DT. Análise do perfil do serviço de Fisioterapia do setor secundário do SUS de Juiz de Fora/MG [Trabalho de Conclusão de Curso]: Faculdade de Medicina da Universidade Federal de Juiz de Fora; 2008.

19. Fengler VZ. Avaliação da pressão perineal em mulheres pós-menopáusicas do município de Catuípe/RS [TCC]. Governo do Município de Criciúma: Universidade Regional do Noroeste do Estado do Rio Grande do Sul; 2012.

20. Pompeu SMAA, Pompeu JE, Moral C, Yumi É. Perfil funcional dos pacientes atendidos no setor de fisioterapia neurológica do Promove São Camilo:[revisão]. Mundo saude. 2010;34(2):218-24.

21. Mazzola D, Polese JC, Schuster RC, Oliveira SG. Perfil dos pacientes acometidos por acidente vascular encefálico assistidos na clínica de fisioterapia neurológica da Universidade de Passo Fundo. Rev bras educ méd. 2007;20(1):22-7. 
Artigo Original

Saúde Funcional

22. Soares PN. Estudo epidemiológico dos pacientes atendido no setor de Fisioterapia Pneumofuncional da clínica de Fisioterapia da UNIPAV. XI Encontro Latino Americano de Iniciação Científica e VII Encontro Latino Americano de PósGraduação - Universidade do Vale do Paraíba; 2006.

23. Santana CMJ. Perfil dos pacientes atendidos no setor de Fisioterapia respiratória da universidade Tiradentes. 15 Simpósio internacional de Fisioterapia Respiratória; 2010. 